\title{
Three dimensions for two
}

\section{Cellular automata make it possible to produce some special solutions of the Ising-lattice problem in three dimensions. Will the hunt for general solutions now begin again?}

THE title "Exact results for two and threedimensional Ising and Potts models"' will excite only a small community of physicists, but for them it will be eye-catching in the highest degree. For is this not the solution to a problem on which a host of people have broken their heads in the past forty years? That, certainly, is how it seems but the title is a little misleading, almost like the headlines with which popular newspapers try to capture readers when the flow of news is slack. And if the title, that of a paper by Eytan Domany from the Weizmann Institute in Rehovot, Israel, but writing from Stanford University (Phys. Rev.Lett. 52, 871; 12 March 1984), had more properly read "Some exact results. ...", whoever would have been sitting on the edge of his chair?

But even if the text of Domany's paper is something of a letdown, it is an interesting and potentially important development in the solution of what is probably best known as the Onsager problem. The problem was first made interesting (in the sense of being made tractable) by Lars Onsager in the 1940s. The Ising lattice, so called since the 1920 s, is the simplest model there could be of a ferromagnetic material as well as of many other kinds of systems capable of order-disorder transitions.

The problem is to calculate the thermodynamic properties of a lattice in which each vertex is occupied by a physical variable which can have one of only two values, say -1 and +1 , and suppose that only interactions between nearest neighbours in the lattice are significant. The energy of interaction between two neighbouring sites will depend only on the values assumed by the two sites. If the variables represent electron spins, the model may describe a ferromagnetic material. If they represent electric dipoles, the lattice will stand for a ferroelectric material (which is, of course, an ordinary dielectric above some critical temperature). If the physical variable represents one or other of two different kinds of atoms, the system likens that of binary alloys such as $\beta$-brass whose mechanical properties depend critically on whether there is or is not long-range order in the system.

Onsager's achievement was to demonstrate that it is possible to obtain exact solutions for the two-dimensional Ising lattice, at least with the help of seemingly undemanding assumptions such as that the properties of a finite piece of lattice are those of an infinite lattice in which the finite piece repeats itself periodically. The importance of Onsager's innovation, essentially a neat piece of algebra, was that it was the first exact calculation of the thermodynamic properties of a system in which phase transitions might be expected. Earlier treatments of the thermodynamics of phase transitions, that of Landau for example, entailed expansions of the free energy of each of two phases in terms of some small quantity (say the difference be tween temperature and transition temperature) and were thus unable to describe the behaviour of thermodynamic quantities arbitrarily close to the transition.

So, forty years ago, it seemed natural to suppose that the problem of phase transitions had been solved once and for all. Onsager's original solution was for a twodimensional square lattice. $\mathrm{He}$ and $\mathrm{G}$. $\mathrm{H}$. Wannier soon afterwards provided a solution for a triangular lattice and, interestingly, showed that that was the equivalent to the solution for a hexagonal lattice obtained by drawing a line perpendicular to each triangular side through its mid-point. (The thermodynamic properties of the "inverse" lattice are those of the original provided that the temperature is inverted by the critical temperature.)

In reality, experience has been frustrating. A variety of two-dimensional lattices have been solved by variants of Onsager's technique or by other means. The model has been generalized in a variety of ways the Potts model is, for example, the generalization in which each vertex of a lattice is occupied by some analogue of spin that can assume more than two values. The Ising lattice has also, quite unexpectedly, been the starting point for A. H. Wilson's demonstration that the renormalization of a gauge field theory can be represented by the calculations of the properties of a suitably chosen lattice. But the threedimensional Ising lattice has never been exactly solved by Onsager's method or any other. People have had to be satisfied with numerical calculations (which nevertheless show that, for example, phase transitions on a three-dimensional lattice do involve a finite change of heat content, as observed when cooling iron through its Curie temperature). It has even been suggested that the three-dimensional problem is inherently insoluble.

That it should be difficult is easily appreciated. To calculate the thermodynamic properties of even a model system, it is necessary to enumerate all possible particular values of the energy. The next step is to construct the partition functions for the system, the sum over all possible energy states $E_{\mathrm{j}}$ of the quantity $N_{\mathrm{j}} \exp \left(E_{\mathrm{j}} / k T\right)$ where $k$ is Boltzmann's constant, $T$ the temperature and $N_{\mathrm{j}}$ is the number of configurations with energy $E_{\mathrm{j}}$. The thermodynamic quantities are then simply derived - the entropy, for example, is simply proportional to the logarithm of the partition function. The snag is that evaluating the quantities $N_{\mathrm{j}}$ is an awkward combinatorial calculation. Onsager's twodimensional trick was to make the partition function factorize.

So how can Domany have made progress even with some particular examples of a three-dimensional lattice? His trick is to combine the known solution of a twodimensional hexagonal lattice with another combinatorial kind of calculation, the technique of cellular automata popularized (after J. von Neumann) by Stephen Wolfram (see Nature 305,$469 ; 1983$ ). That is a means by which the evolution in "time" of an array of objects may be simulated by machine once rules have been specified for the dependence of the members of one "generation" on those of the predecessor generation.

The advantage of starting with a hexagonal Ising lattice is that when the interactions between neighbours are such that the whole system is ferromagnetic, the lattice can be considered as if it were two interpenetrating triangular lattices inverted with respect to each other. The rules for the time evolution of such a system considered as a cellular automaton make it possible to regard each successive "generation" in the calculation as another layer of a threedimensional lattice. But the outcome is, as followers of Wolfram might expect, not a three-dimensional lattice of identical planes but one in which hexagonal and triangular arrays alternate as the structure known as hexagonal close-packed.

The same trick works with the Potts model - starting with a hexagonal lattice in two dimensions, Domany is able to generate a solution for a hexagonal closepacked lattice in three dimensions. For the time being, the interest of much of this argument is that it should be possible to ground at least some of the conjectures based on numerical calculations in analytical results. But nobody should be surprised if the old Adam - the hunt for a general three-dimensional solution - does not begin all over again.

John Maddox 